Ciência Florestal, Santa Maria, v. 23, n. 3, p. 357-368, jul.-set., 2013

ISSN 0103-9954

\title{
LODO DO CURTIMENTO E SUA INFLUÊNCIA NA PRODUÇÃO DE MUDAS DE PARICÁ (Schizolobium amazonicum) E NAS PROPRIEDADES QUÍMICAS DO SOLO
}

\author{
TANNING SLUDGE AND ITS INFLUENCE ON THE PRODUCTION OF SEEDLINGS OF PARICÁ \\ (Schizolobium amazonicum) AND ON CHEMICAL PROPERTIES OF SOIL
}

\author{
Lorena de Souza Tavares ${ }^{1}$ Walcylene Lacerda Matos Pereira Scaramuzza ${ }^{2}$ \\ Oscarlina Lúcia dos Santos Weber ${ }^{2}$ Franciele Caroline de Assis Valadão ${ }^{3}$ Kelly Dayana Benedet Maas ${ }^{4}$
}

\section{RESUMO}

O objetivo deste estudo foi verificar a influência do lodo do curtimento nas propriedades químicas do solo e nas características morfológicas e nutricionais de mudas de paricá. O experimento foi realizado em casa de vegetação em delineamento inteiramente casualizado, em cinco repetições, tendo como substrato um cambissolo húmico, o qual foi caracterizado química e granulometricamente; de posse desses resultados o solo foi corrigido previamente com calcário e NPK. Após 100 dias de observação foram analisadas as variáveis químicas do solo ( $\mathrm{pH}, \mathrm{H}+\mathrm{Al}, \mathrm{Al}^{3+}, \mathrm{Ca}, \mathrm{Mg}, \mathrm{P}, \mathrm{K}, \mathrm{Na}, \mathrm{S}, \mathrm{Cr}^{3+}, \mathrm{CO}, \mathrm{SB}, \mathrm{CTC}, \mathrm{V}, \mathrm{RAS}$ e PST), a morfologia da planta [altura $(\mathrm{H})$, diâmetro do caule $(\mathrm{DC})$, número de folhas completas, comprimento da raiz principal (CR) e biomassa seca], bem como as concentrações de $\mathrm{N}, \mathrm{P}, \mathrm{K}, \mathrm{Ca}, \mathrm{Mg}, \mathrm{S}, \mathrm{Cr}^{3+}$ e Na na planta. O lodo de curtimento foi utilizado como complemento nutricional nas doses de 0,$0 ; 1,5 ; 3,0 ; 4,5$ e $6,0 \mathrm{~g} \mathrm{~kg}^{-1}$. O lodo de curtimento tem potencial como corretivo de acidez do solo, com aumento pouco expressivo dos indicadores de salinidade, sodicidade e do teor de $\mathrm{Cr}^{3+}$ disponível. No entanto, o uso do lodo não afetou as características morfológicas das mudas de paricá, porém, propiciou aumento nos teores $\mathrm{K}$ e $\mathrm{S}$ na parte aérea e de $\mathrm{Na}$ e $\mathrm{S}$ na raiz. Os maiores teores de nutrientes no paricá foram obtidos com $6,0 \mathrm{~g} \mathrm{~kg}^{-1} \mathrm{de}_{\text {lodo de }}$ curtimento, contudo, não foi possível otimizar uma dose para a máxima produtividade de mudas de paricá com as doses utilizadas.

Palavras-chave: resíduo industrial; cromo; nutrição florestal; salinização do solo.

\begin{abstract}
The objective of this study was to assess the influence of the tanning sludge in soil chemical properties and the morphological and nutritional of paricá seedlings. The experiment was conducted in a greenhouse in a completely randomized design with five replicates, having as a substrate humic cambisol, which was characterized chemically and granulometrically; in possession of these results, the soil was previously corrected with lime and NPK. After 100 days of observation, it was analyzed the soil chemical variables (pH, H $+\mathrm{Al}, \mathrm{Al}^{3+}, \mathrm{Ca}, \mathrm{Mg}, \mathrm{P}, \mathrm{K}, \mathrm{Na}, \mathrm{S}, \mathrm{Cr}^{3+}, \mathrm{CO}, \mathrm{SB}, \mathrm{CEC}, \mathrm{V}, \mathrm{RAS}$ and PST), the morphology plant [height $(\mathrm{H})$, stem diameter (DC), the number of full leaves, the main root length (CR) and dry biomass], and the concentrations of $\mathrm{N}, \mathrm{P}, \mathrm{K}, \mathrm{Ca}, \mathrm{Mg}, \mathrm{S}, \mathrm{Cr}^{3+}$ and $\mathrm{Na}$ in the plant. The tanning sludge was used as a nutritional supplement in doses of $0.0,1.5,3.0,4.5$ and $6.0 \mathrm{~g} \mathrm{~kg}^{-1}$. The tanning sludge has potential as a corrective

1 Engenheira Florestal, Doutoranda do Programa de Pós-Graduação em Agricultura Tropical, Universidade Federal do Mato Grosso, Professora do Instituto Federal de Rondônia, Rua Amazonas, 151, Jardim dos Migrantes, CEP 76900-730, Ji-Paraná (RO). lorena.tavares@ifro.edu.br

2 Engenheira Agrônoma, Dra ${ }^{\mathrm{a}}$, Professora Adjunta do Departamento de Solos e Engenharia Rural, Faculdade de Medicina Veterinária, Universidade Federal do Mato Grosso, Av. Fernando Correa da Costa, s/n, Coxipó, CEP 78060-900, Cuiabá (MT).walcylene@ufmt.br / oscsanwb@cpd.ufmt.br

3 Engenheira Agrônoma, Msc., Professora do Instituto Federal de Mato Grosso, Rod. MT235, Km 12, Zona Rural, Caixa Postal 100, CEP 78360-000, Campo Novo do Parecis (MT). franciele.valadão@cnp.ifmt.edu.br

4 Tecnóloga Ambiental, Doutoranda do Programa de Pós-Graduação em Agricultura Tropical, Universidade Federal de Mato Grosso, Av. Fernando Correia da Costa, s/n, Coxipó, CEP 78060-900, Cuiabá (MT). kelly bmaas@hotmail.com

Recebido para publicação em 26/11/2010 e aceito em 21/05/2012
\end{abstract}


of soil acidity, with little significant increase in indicators of salinity, sodicity and $\mathrm{Cr}^{3+}$ content available. However, the use of sludge did not affect the morphological characteristics of seedlings of paricá, but induced an increase in levels $\mathrm{K}$ and $\mathrm{S}$ in the shoot and $\mathrm{Na}$ and $\mathrm{S}$ in the root. The greatest concentration of nutrients in paricá were obtained with $6.0 \mathrm{~g} \mathrm{~kg}^{-1}$ of tanning sludge; however, was not possible to optimize the dose for a maximum productivity of seedlings of paricá with the treatments used.

Keywords: industrial waste; chromium; forest nutrition; soil salinization.

\section{INTRODUÇÃO}

O paricá (Schizolobium amazonicum) é uma espécie florestal nativa encontrada nos estados do Amazonas, Pará, Mato Grosso e Rondônia, indicada para plantios comerciais, sistemas agroflorestais e reflorestamento de áreas degradadas, devido ao seu rápido crescimento e alto valor comercial. No mesmo ritmo acelerado de crescimento, estudos como os de Marques et al. (2004) e Bressiani (2010) evidenciaram ser o paricá uma espécie com alta exigência nutricional, indicando a necessidade de alta disponibilidade de nutrientes para expressar todo seu potencial produtivo.

Alternativa aos fertilizantes comerciais, a utilização de resíduos provindos de sistemas agrícolas ou industriais, podem gerar resultados convenientes, tanto do ponto de vista econômico como ambiental. Neste contexto, encontram-se os resíduos da indústria coureira, que, ao serem despejados na natureza de forma inadequada, são potencialmente tóxicos por possuírem, principalmente, alta concentração de cromo e sódio, como é o caso do lodo de curtimento (CASTILHO et al., 1999).

O lodo do curtimento é gerado no fim do processo produtivo do couro, em que se utiliza o metal pesado cromo na forma trivalente $\left(\mathrm{Cr}^{3+}\right)$ como agente curtidor, a fim de aumentar a estabilidade do sistema colágeno, tornando o couro imputrescível ao ataque dos microrganismos (MARTINES, 2005).

Dessa forma, o lodo do curtimento possui componentes orgânicos de origem animal misturados com sais inorgânicos, e alguns desses componentes são nutrientes para plantas e microrganismos, além de agentes corretivos da acidez do solo, devido às quantidades significativas de carbonatos, hidróxidos de cálcio e sulfetos (SELBACH et al., 1991), que não foram totalmente removidos nas operações de desencalagem, e aos sais de cromo, que possuem basicidade elevada (PRIEBE, 2005).

Em alguns trabalhos é possível verificar a viabilidade e eficiência deste resíduo na agricultura, tanto na elevação do $\mathrm{pH}$ do solo quanto para o fornecimento de alguns nutrientes (CASTILHOS et al., 1999; MARTINES, 2005; ARAÚJO et al., 2006; TEIXEIRA et al., 2006; SOUZA et al., 2006; SOUZA, 2009). Pesquisas envolvendo o cultivo de espécies alimentares com o uso do lodo de curtimento também têm sido desenvolvidas (COSTA et al., 2001; KONRAD e CASTILHOS, 2002; FERREIRA et al., 2003; ARAÚJO et al., 2008).

Todavia, a preocupação com relação às quantidades cumulativas de substâncias químicas do lodo nos alimentos ainda persiste, o que poderia trazer riscos à saúde humana e ao meio ambiente. Assim, o uso do lodo na produção de mudas de espécies florestais consistiria em um modo mais seguro e racional de aproveitamento desse resíduo, considerando que o cromo ficaria retido na madeira, não oferencendo ameaça de liberação, além de colaborar para a redução do uso de fertilizantes comerciais.

Diante dessa possibilidade, o presente trabalho teve como objetivo verificar a influência do lodo do curtimento nos atributos do solo, nas características morfológicas e nutricionais de mudas de paricá.

\section{MATERIAL E MÉTODOS}

O experimento foi realizado na casa de vegetação da Faculdade de Agronomia e Medicina Veterinária (FAMEV) da Universidade Federal de Mato Grosso (UFMT), em Cuiabá-MT, entre os meses de março e julho de 2009, coincidindo com o final do período chuvoso. Durante o experimento, foram registradas as temperaturas médias, com máxima de $41^{\circ} \mathrm{C}$, mínima de $22^{\circ} \mathrm{C}$ e umidade relativa do ar de $56 \%$.

Foiutilizado como substrato um Cambissolo Húmico, coletado na camada de 0 a $0,20 \mathrm{~m}$, caracterizado química e granulometricamente conforme EMBRAPA (2009) (Tabela 1). Foram determinados o pH: obtido por $\mathrm{CaCl}_{2} 0,01 \mathrm{~mol} \mathrm{~L}^{-1}$ na proporção 1:2,5 por potenciometria; $\mathrm{Ca}, \mathrm{Mg}$ e $\mathrm{Al}$ trocáveis: extraídos por $\mathrm{KCl} 1 \mathrm{~mol} \mathrm{~L}^{-1}$, determinados, respectivamente, por titulação compleximétrica e de neu- 
tralização; $\mathrm{H}+\mathrm{Al}$ : extraído por acetato de cálcio, determinado por neutralização; $\mathrm{K}$ e Na trocáveis e $\mathrm{P}$ e Cr disponíveis: extraídos por Mehlich 1, determinados, respectivamente, por fotometria de chama, colorimetria e absorção atômica; N: extraído por digestão sulfúrica Kjedahl, determinado por titulação de neutralização; S: extraído por fosfato de cálcio, determinado por turbidimetria; e a textura pelo método do densímetro em $\mathrm{NaOH} 0,5 \mathrm{~mol} \mathrm{~L}^{-1}$.

O resíduo lodo do curtimento foi proveniente do Curtume Durli Ltda., o qual foi analisado quanto aos teores totais dos elementos químicos, conforme metodologia descrita pelo Ministério da Agricultura, Pecuária e Abastecimento - MAPA (1988), (Tabela 2).

O delineamento experimental foi inteiramente casualizado com cinco tratamentos e cinco repetições, sendo os tratamentos constituídos pelas doses crescentes de lodo de curtimento $(0,0 ; 1,5$; 3,0; 4,5 e 6,0 $\mathrm{g} \mathrm{kg}^{-1}$ ) em solo previamente corrigido com NPK+calcário, sendo que cada unidade experimental foi composta por um saco plástico com capacidade para de $5 \mathrm{dm}^{3}$, sendo preenchido com $3,5 \mathrm{~kg}$ de solo previamente peneirado em malha de
$2 \mathrm{~mm}$ e seco ao ar (terra fina seca ao ar - TFSA), e uma planta.

A irrigação do substrato seguiu o critério de manter a capacidade máxima de retenção de água a $60 \%$ por meio de aferições diárias por pesagem. Após a análise química do solo e do lodo, foi observada a necessidade de complementação da adubação NPK, com base nas recomendações de adubação. A recomendação das adubações de $\mathrm{N}$ e $\mathrm{K}$ foi realizada de acordo com Ribeiro et al. (1999), e a adubação de fósforo (P) foi feita segundo Alvarez V. et al. (2000). A adição do calcário teve por finalidade elevar a saturação por bases a $50 \%$, baseando-se nas recomendações feitas para outras espécies florestais nativas como a seringueira (RIBEIRO et al., 1999) e ipê (CRUZ et al., 2004), tendo em vista que, para o paricá, ainda não há recomendação.

As sementes de paricá foram submetidas à quebra de dormência, utilizando o método de escarificação mecânica com esmerilamento na parte oposta à micrópila (ROSSA, 2008), sendo posteriormente colocadas para germinar diretamente nos sacos plásticos.

TABELA 1: Caracterização química e granulométrica do solo utilizado como substrato.

TABLE 1: Texture and chemical analysis of the soil used as substrate.

\begin{tabular}{|c|c|c|c|c|c|c|c|c|c|}
\hline $\mathrm{pH}$ & $\mathrm{P}$ & $\mathrm{K}$ & $\mathrm{Na}$ & $\mathrm{S}$ & $\mathrm{Cr}$ & $\mathrm{MO}$ & $\mathrm{C}$ & $\mathrm{V}$ & $\mathrm{M}$ \\
\hline ---- & & ---- & $-m g$ & --- & --- & \multicolumn{2}{|c|}{--- $\mathrm{g} \mathrm{dm}^{-3}$--- } & \multicolumn{2}{|c|}{---- \% ---- } \\
\hline 4,4 & 2,1 & 30 & 2,0 & 6,8 & 2,9 & 25,5 & 14,82 & 22,7 & 21,2 \\
\hline \multirow[t]{2}{*}{$\mathrm{H}+\mathrm{Al}$} & $\mathrm{Al}$ & $\mathrm{H}$ & $\mathrm{Ca}$ & $\mathrm{Mg}$ & CTC & SB & areia & silte & argila \\
\hline & & & $\mathrm{cmo}$ & 3 & & & -- & $\mathrm{g} \mathrm{kg}$ & ---- \\
\hline 5,3 & 0,4 & 4,9 & 0,9 & 0,6 & 6,9 & 1,6 & 616 & 67 & 317 \\
\hline
\end{tabular}

Em que: pH: potencial hidrogeniônico; P: fósforo; K: potássio; Na: sódio; S: enxofre; Cr: cromo; MO: matéria orgânica; C: carbono; V: saturação por bases; M: saturação por alumínio; $\mathrm{H}+\mathrm{Al}$ : acidez potencial; Al: alumínio; H: acidez ativa; Ca: cálcio; Mg: magnésio; CTC: capacidade de troca de cátions; SB: soma de bases.

TABELA 2: Caracterização química do lodo de curtimento.

TABLE 2: Chemical analysis of tannery sludge.

\begin{tabular}{|c|c|c|c|c|c|}
\hline $\mathrm{pH} \mathrm{CaCl}{ }_{2}$ & $\mathrm{~N}$ & $P$ & K & $\mathrm{Ca}$ & $\mathrm{Mg}$ \\
\hline 8,6 & 20,4 & 6,6 & 2,2 & 68,0 & 4,8 \\
\hline $\mathrm{CE}$ & $\mathrm{Na}$ & $\mathrm{S}$ & $\mathrm{Cr}$ & $\mathrm{CO}$ & $\mathrm{MO}$ \\
\hline $\mathrm{dS} \mathrm{m}^{-1}$ & & 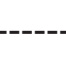 & l) $\mathrm{g} \mathrm{d}$ & $\cdots$ & \\
\hline 1,81 & 26,0 & 9,7 & 19,82 & 305,6 & 526,9 \\
\hline
\end{tabular}

Em que: pH: potencial hidrogeniônico em cloreto de cálcio; N: nitrogênio; P: fósforo; K: potássio; Ca: cálcio; Mg: magnésio; CE: condutividade elétrica; Na: sódio; S: enxofre; Cr: cromo; CO: carbono orgânico; MO: matéria orgânica. 
Após 100 dias de observação, foram analisadas as variáveis químicas do solo $\mathrm{pH}, \mathrm{H}+\mathrm{Al}_{1} \mathrm{Al}^{3+}$, $\mathrm{Ca}, \mathrm{Mg}, \mathrm{P}, \mathrm{K}, \mathrm{Na}, \mathrm{S}, \mathrm{Cr}^{3+}$ e Carbono Orgânico (CO), determinadas segundo metodologia da EMBRAPA (2009). A soma de bases (SB), a capacidade de troca catiônica (CTC), a saturação por alumínio e por bases $(\mathrm{V} \%)$ foram obtidos por meio de cálculos. A condutividade elétrica (CE) foi determinada pela metodologia de Camargo et al. (1986), e o CO foi quantificado pela metodologia de Yeomans e Bremer (1988). Também foram verificados a razão de adsorção de sódio (RAS) e o percentual de sódio trocável (PST) (CAMPOS et al., 2009).

As mudas de paricá foram avaliadas quanto aos aspectos morfológicos: altura de plantas $(\mathrm{H})$ $(\mathrm{cm})$; diâmetro do caule (DC) $(\mathrm{mm})$; número de folhas completas; e comprimento da raiz principal (CR) $(\mathrm{cm})$. A altura foi obtida a partir da medição do nível do substrato até a inserção da última folha da planta (broto), com a utilização de uma fita métrica; o diâmetro do caule foi mensurado com o auxílio de um paquímetro digital de precisão $0,01 \mathrm{~mm}$ na altura de $5 \mathrm{~cm}$ do substrato; o número de folhas foi obtido pela contagem de folhas completas; e o comprimento da raiz principal foi conseguido com medições por meio de uma régua. Após a coleta, as partes das plantas foram acondicionadas em sacos de papel e levadas à estufa de ventilação forçada regulada a $65^{\circ} \mathrm{C}$ para a secagem até atingirem peso constante. As amostras foram pesadas em balanças eletrônicas com precisão de quatro casas após a vírgula, quantificando-as em biomassa seca da parte aérea (MSPA) (g) que envolvia folhas e caules, biomassa seca da raiz (MSRA) (g) e biomassa seca total (MST) (g), que é a somatória de todas as partes das plantas citadas anteriormente, além das relações entre as variáveis MSPA/MSRA; H/DC; H/MSPA e o índice de qualidade de Dickson (IQD), calculado por meio da fórmula (DICKSON et al., 1960): IQD = PMST(g)/ $[\mathrm{H}(\mathrm{cm}) / \mathrm{DC}(\mathrm{mm})]+[\mathrm{MSPA}(\mathrm{g}) / \operatorname{MSRA}(\mathrm{g})]$. Em seguida foram determinadas as concentrações de N, $\mathrm{P}$, $\mathrm{K}, \mathrm{Ca}, \mathrm{Mg}, \mathrm{S}, \mathrm{Cr}^{3+}$ e Na na planta, conforme métodos descritos em EMBRAPA (2009).

Os resultados foram submetidos ao teste de normalidade Ryan-Joyner $(\mathrm{p}>0,10)$ e Análise de Variância. No caso de significância do Teste $\mathrm{F}$ $(\mathrm{p}<0,05)$ procedeu-se à Análise de Regressão, sendo considerado adequado o modelo com distribuição normal dos resíduos, variância constante, coeficiente de determinação maior que 0,50 e parâmetros da equação significativos $(p<0,05)$. Para análises estatísticas e elaboração dos gráficos, foi utilizado o apli- cativo computacional Minitab 15.

\section{RESULTADOS E DISCUSSÃO}

\section{Alterações nos atributos químicos do solo}

Não foram observados efeitos significativos das doses de lodo de curtimento nos teores de N, $\mathrm{P}, \mathrm{K}, \mathrm{Mg}$ e Carbono Orgânico (CO) no solo. A não significância de N, P, K e Mg possivelmente ocorreram pelas baixas concentrações desses elementos no lodo, conforme evidenciado na Tabela 2. Essa baixa concentração no lodo também foi observada por autores como Costa et al. (2001), Konrad e Castilhos (2002) e Ferreira et al. (2003). Quanto ao CO, não foi observada diferença entre os tratamentos após 100 dias, provavelmente esse fato tenha ocorrido pela rápida mineralização do $\mathrm{CO}$ ao longo do tempo, haja vista que a temperatura contribuiu para a alta atividade biológica, atingindo valores estáveis. Concordando com estes resultados está Martines (2006), que em seu trabalho verificou que a atividade microbiana foi mais intensa nos primeiros 20 dias de incubação, decrescendo posteriormente, pois os microrganismos atuam com maior intensidade na presença de maior disponibilidade de material orgânico; e Costa et al. (2008) que confirmam a relação do aumento da temperatura no solo com a intensidade da atividade microbiana.

Por outro lado, foi possível verificar no solo aumento do $\mathrm{pH}$, redução da acidez potencial $(\mathrm{H}+\mathrm{Al})$, do alumínio $\left(\mathrm{Al}^{3+}\right)$ e da saturação por alumínio $(\mathrm{m} \%)$ com a utilização do lodo de curtimento (Figura 1).

Os valores de $\mathrm{pH}$ do solo (Figura 1A) ajustaram-se ao modelo quadrático, com ponto máximo da curva na dose de $5,66 \mathrm{~g} \mathrm{~kg}^{-1}$ de lodo, que correspondeu ao $\mathrm{pH}$ de 5,85; enquanto que para a acidez potencial (Figura 1B) houve redução linear de acordo com aumento das doses de lodo. O lodo teve efeito na neutralização do $\mathrm{Al}^{3+}$ (Figura $1 \mathrm{C}$ ), pois, no tratamento sem lodo, o efeito do calcário elevou o pH inicial do solo de 4,4 para 5,01 e reduziu o $\mathrm{Al}^{3+}$ em $0,1 \mathrm{cmol}_{\mathrm{c}} \mathrm{kg}^{-1}$, enquanto com a aplicação do lodo houve neutralização total.

A redução dos teores de $\mathrm{Al}^{3+}$ foi semelhante ao de $\mathrm{m} \%$ (Figura 1D), ajuste quadrático, no entanto, este último é considerado um dos melhores índices para estimar o nível de toxidez de $\mathrm{Al}^{3+}$ nos solos tropicais (EMBRAPA, 2004), pois leva em conta à proporção que este elemento ocupa na CTC efetiva. Assim, a saturação por alumínio decresceu de 9,78\% 

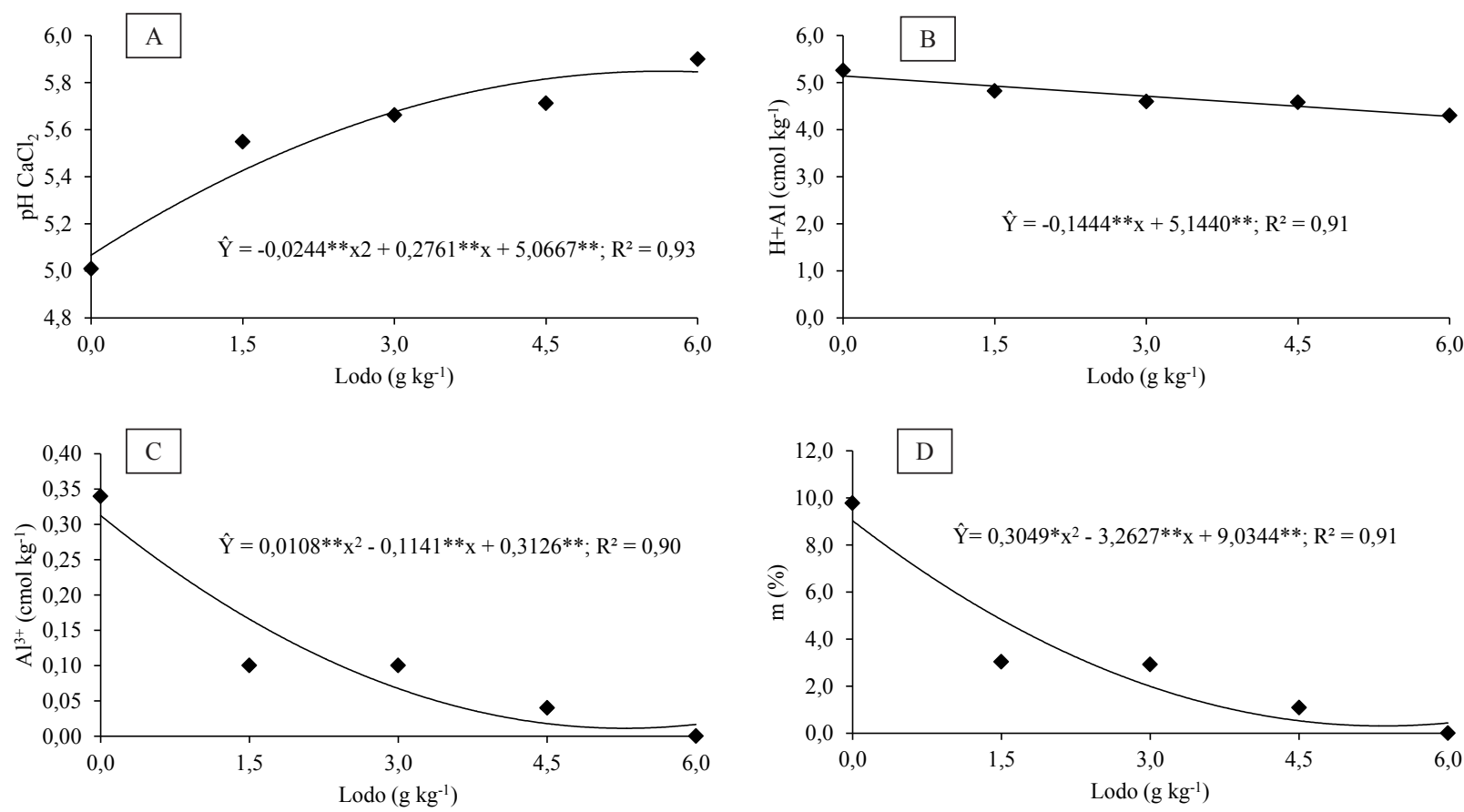

FIGURA 1: $\mathrm{pH}$ em cloreto de cálcio (A), acidez potencial - $\mathrm{H}+\mathrm{Al}(\mathrm{B})$, teor de alumínio - $\mathrm{Al}^{+3}(\mathrm{C})$ e saturação por alumínio - $\mathrm{m}$ (D) no solo em função das doses de lodo do curtimento. ${ }^{* *}$ Coeficientes significativos $\mathrm{p}<0,01 ;{ }^{*}$ Coeficientes significativos $\mathrm{p}<0,05$.

FIGURE 1: $\mathrm{pH}$ in calcium chloride (A), potential acidity $-\mathrm{H}+\mathrm{Al}(\mathrm{B})$, aluminum content $-\mathrm{Al}^{+3}(\mathrm{C})$ and aluminum saturation - $m(D)$ in soil as a function of doses of the tanning sludge. ** Significant coefficients $\mathrm{p}<0.01 ; *$ Significant coefficients $\mathrm{p}<0.05$.

no tratamento sem lodo para zero na maior dose de lodo $\left(6,0 \mathrm{~g} \mathrm{~kg}^{-1}\right)$, efeito desejável pela toxicidade do $\mathrm{Al}^{3+}$ ao desenvolvimento das plantas (FOY, 1992; SALVADOR et al., 2000; CRUZ et al., 2008).

Dessa forma, mesmo com aplicação de calcário em todos os tratamentos, o resíduo atuou como corretivo da acidez do solo, conforme também observado por Castilhos et al. (1999), Ferreira et al. (2003) e Martines (2005). Isso é justificado pela presença de carbonatos e hidróxidos de cálcio no lodo, provenientes das etapas de depilação (SELBACH et al., 1991) e aos sais de cromo $\left(\mathrm{Cr}^{3+}\right)$, que apresentam basicidade elevada (PRIEBE, 2005).

Os teores de $\mathrm{Ca}, \mathrm{Na}, \mathrm{Cr}^{3+}$ e V\% aumentaram linearmente com aumento das doses do lodo do curtimento (Figura 2). Isso, possivelmente, deveu-se às etapas anteriores de processamento do couro, principalmente as de ribeira e do caleiro, onde são adicionadas fontes como carbonatos, hidróxidos, sódio e sulfetos (SELBACH et al., 1991), que se encontram na composição do lodo do curtimento como formas residuais.

O teor de Ca no solo (Figura 2A) aumentou
0,64 cmol $\mathrm{kg}^{-1}$ da maior dose de lodo $\left(6,0 \mathrm{~g} \mathrm{~kg}^{-1}\right)$ em comparação com o tratamento sem lodo, sendo os valores encontrados classificados como médios de acordo com Ribeiro et al. (1999). Também foi observado acréscimo no teor de sódio $(\mathrm{Na})$ no solo (Figura 2B) de 5,4 vezes na dose mais elevada de lodo $\left(6,0 \mathrm{~g} \mathrm{~kg}^{-1}\right)$, quando comparado ao tratamento sem lodo. Aumento nos teores de Ca e Na também foram obtidos por Costa et al. (2001), Konrad e Castilhos (2002), Ferreira et al. (2003), Teixeira et al. (2006) e Araújo et al. (2008).

Mesmo o resíduo tendo elevada quantidade de $\mathrm{Na}$ em sua composição $\left(26 \mathrm{~g} \mathrm{~kg}^{-1}\right)$, os valores encontrados no solo, na maior dose de lodo, foi de apenas $8,12 \mathrm{mg} \mathrm{kg}^{-1}$ de $\mathrm{Na}$ após o cultivo do paricá, permitindo inferir que esse elemento foi absorvido pela planta. Resultados semelhantes foram obtidos por Ferreira et al. (2003) quando testaram a campo o resíduo, no entanto, atribuíram as baixas quantidades de $\mathrm{Na}$ no solo $\left(5,7 ; 7,6\right.$ e 9,6 $\left.\mathrm{mg} \mathrm{kg}^{-1}\right)$, em relação aos valores da composição química do lodo de curtume, à provável lixiviação desse elemento no período chuvoso. 

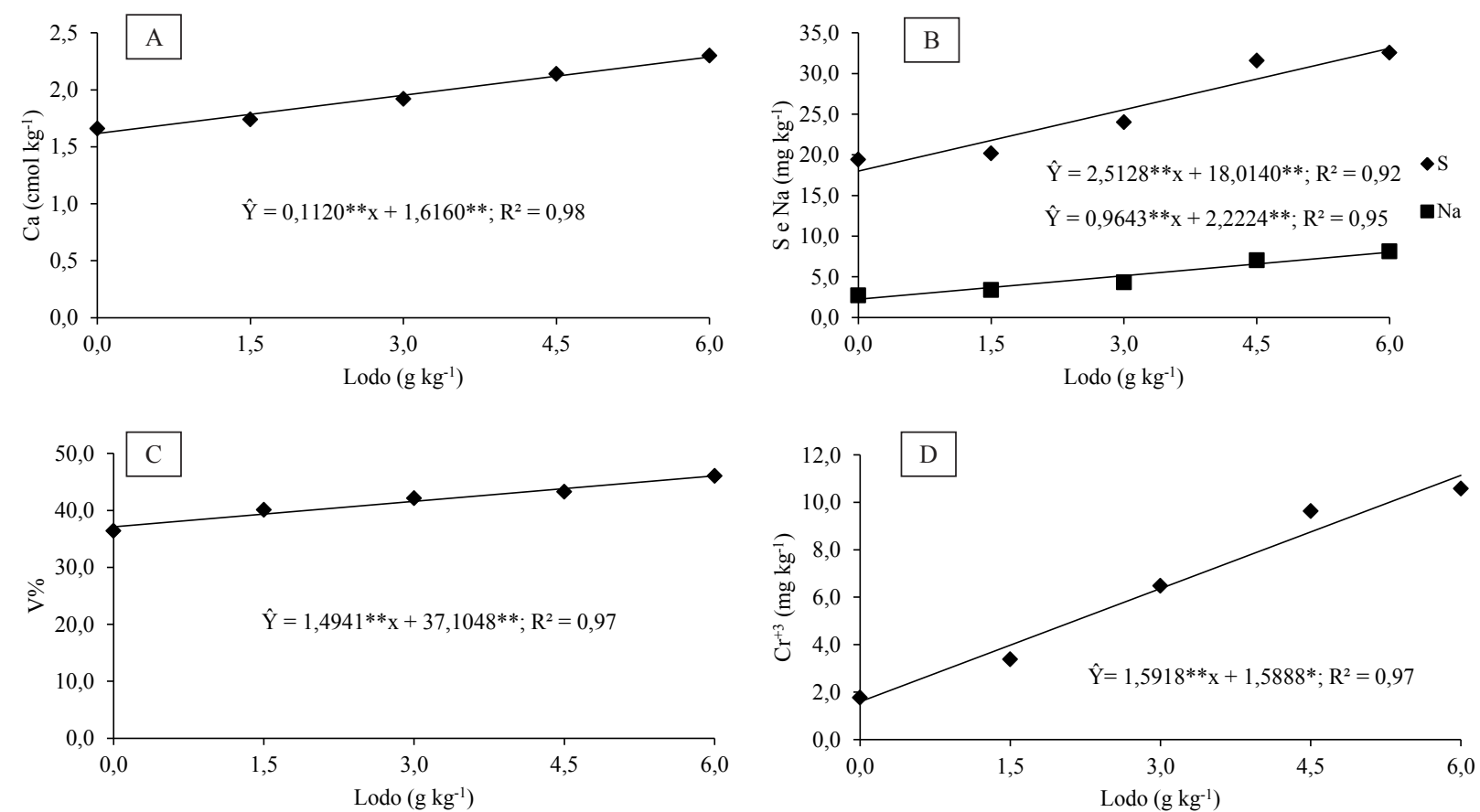

FIGURA 2: Teor de cálcio - Ca (A), sódio - Na e enxofre - S (B), saturação por bases - V (C) e teor de cromo $-\mathrm{Cr}^{+3}(\mathrm{D})$ em função das doses de lodo do curtimento. **Coeficientes significativos $\mathrm{p}<0,01 ; *$ Coeficientes significativos $\mathrm{p}<0,05$.

FIGURE 2: Content of calcium - Ca (A), sodium - Na and sulfur - S (B), saturation - V (C) and chromium $-\mathrm{Cr}^{+3}$ (D) in function of doses of the tanning sludge. ${ }^{* *}$ Significant coefficients $\mathrm{p}<0.01$; *Significant coefficients $\mathrm{p}<0.05$.

Todavia, cabe salientar que aplicações sucessivas desse resíduo no solo implicam em aumentos de $\mathrm{Na}$, cujo acúmulo pode proporcionar impactos negativos sobre o desenvolvimento das culturas e sobre as propriedades físicas do solo (AQUINO NETO e CAMARGO, 2000). Solos com alta concentração de $\mathrm{Na}^{+}$apresentam expansão das entrecamadas de argila provocando o fenômeno de dispersão o que reduz a permeabilidade do solo e causa problemas de drenagem devido ao entupimento dos poros provocada pelas argilas dispersas. Além disto, por estas características do $\mathrm{Na}$, este afeta em maior grau o potencial osmótico do solo limitando o crescimento da planta e a disponibilidade de água a ser consumida, afetando a divisão e o alongamento das células dos vegetais. Porém, a maior dose de lodo do curtimento utilizada correspondeu à adição de $312 \mathrm{~kg} \mathrm{ha}^{-1}$ de $\mathrm{Na}$, não ultrapassando o limite máximo de aplicação anual de $\mathrm{Na}$ em solos, segundo a norma P4.233 da Companhia de Tecnologia de Saneamento Ambiental - CETESB (1999), que é de $1.000 \mathrm{~kg} \mathrm{ha}^{-1}$ para solos argilosos e de $400 \mathrm{~kg} \mathrm{ha}^{-1}$ para arenosos.

Com relação ao teor de $\mathrm{S}$ no solo (Figura 2B), o efeito das doses foi linear, notando-se acréscimos de 1,7 vezes na maior dose em relação à menor dose. Esse aumento ocorreu, principalmente, em razão das quantidades significativas desse elemento na composição do resíduo, devido aos resquícios de sulfatos e sulfetos adicionados no processo de curtimento. Sendo também influenciado pelo aumento do $\mathrm{pH}$, que atua diretamente na mineralização do $\mathrm{S}$, quando próximo da neutralidade (RISTHER, 2009).

Para a saturação por bases (V\%) (Figura 2C), o aumento ocorreu devido à elevada presença de bases, em especial o $\mathrm{Ca}$, que ocorreu em maior quantidade. A saturação por bases encontrada neste trabalho propiciou bom desenvolvimento às mudas de paricá, obtendo na maior dose $\left(6,0 \mathrm{~g} \mathrm{~kg}^{-1}\right)$ saturação de $46 \%$, sendo enquadrada na classificação como média, de acordo com Ribeiro et al. (1999). Da mesma forma, Souza et al. (2008) observaram que as melhores mudas da espécie arbórea Machaerium nictitans, da família das leguminosas, 
desenvolveram-se melhor na saturação por bases de 40 a $70 \%$ em diferentes solos.

Para o teor de $\mathrm{Cr}^{3+}$ no solo (Figura 2D), houve aumento de forma linear em relação às doses de lodo, devido a sua composição proporcionar considerável quantidade desse elemento, na forma de $\mathrm{Cr}^{3+}$, fato verificado também por Costa et al. (2001) e Ferreira et al. (2003). Na maior dose $\left(6,0 \mathrm{~g} \mathrm{~kg}^{-1}\right)$ houve incorporação de aproximadamente $118,9 \mathrm{~g} \mathrm{~kg}^{-1}$ e foram recuperados menos de $10 \%$ do $\mathrm{Cr}^{3+}$ adicionado na análise química, ou seja, estava prontamente disponível. Possivelmente, isso tenha ocorrido porque o $\mathrm{Cr}^{3+}$, em solos com $\mathrm{pH}$ acima de 5,0 é precipitado em formas insolúveis de $\mathrm{Cr}\left(\mathrm{OH}_{3}\right)$. $\mathrm{nH}_{2} \mathrm{O}$ no solo (BARTLETT e KIMBLE, 1976; KONRAD e CASTILHOS, 2002), assim, não sendo tóxicos às plantas, pois a toxicidade só acontece em meios muito ácidos (MCGRANTH e SMITH, 1997). Ressalta-se, ainda, que as quantidades adicionadas do metal nos tratamentos foram menores que o limite estabelecido pela CETESB (2005), que adotam como referência teor de 150 a $400 \mathrm{mg} \mathrm{kg}^{-1} \mathrm{de}$
$\mathrm{Cr}^{3+}$ no solo.

Como critério para classificar um solo como salino ou sódico, utiliza-se o percentual de sódio trocável (PST), a razão de adsorção de sódio (RAS), o $\mathrm{pH}$ e a condutividade elétrica (CE) (MARTINES, 2005). Diante disso, foi diagnosticado que essas variáveis aumentaram linearmente de acordo com as doses (Figura 3), todavia, nenhuma ultrapassou os índices permitidos. O PST (Figura 3A) foi considerado baixo, pois os resultados não ultrapassaram $15 \%$, limite usado para classificar solos sódicos, assim como a RAS (Figura 3B), que atingiu nível muito baixo $(<10)$, sendo classificada como risco baixo de sodicidade, não interferindo na infiltração. A CE (Figura 3C) do solo permaneceu abaixo de $4 \mathrm{dS} \mathrm{m}^{-1}$, sendo, portanto, o solo classificado como não sódico e não salino, de acordo com Richards (1954).

A condutividade elétrica aumentou devido à presença de sais no lodo de curtimento, principalmente pela elevada concentração de $\mathrm{Na}$ contida no resíduo. Estes resultados estão em concordân-
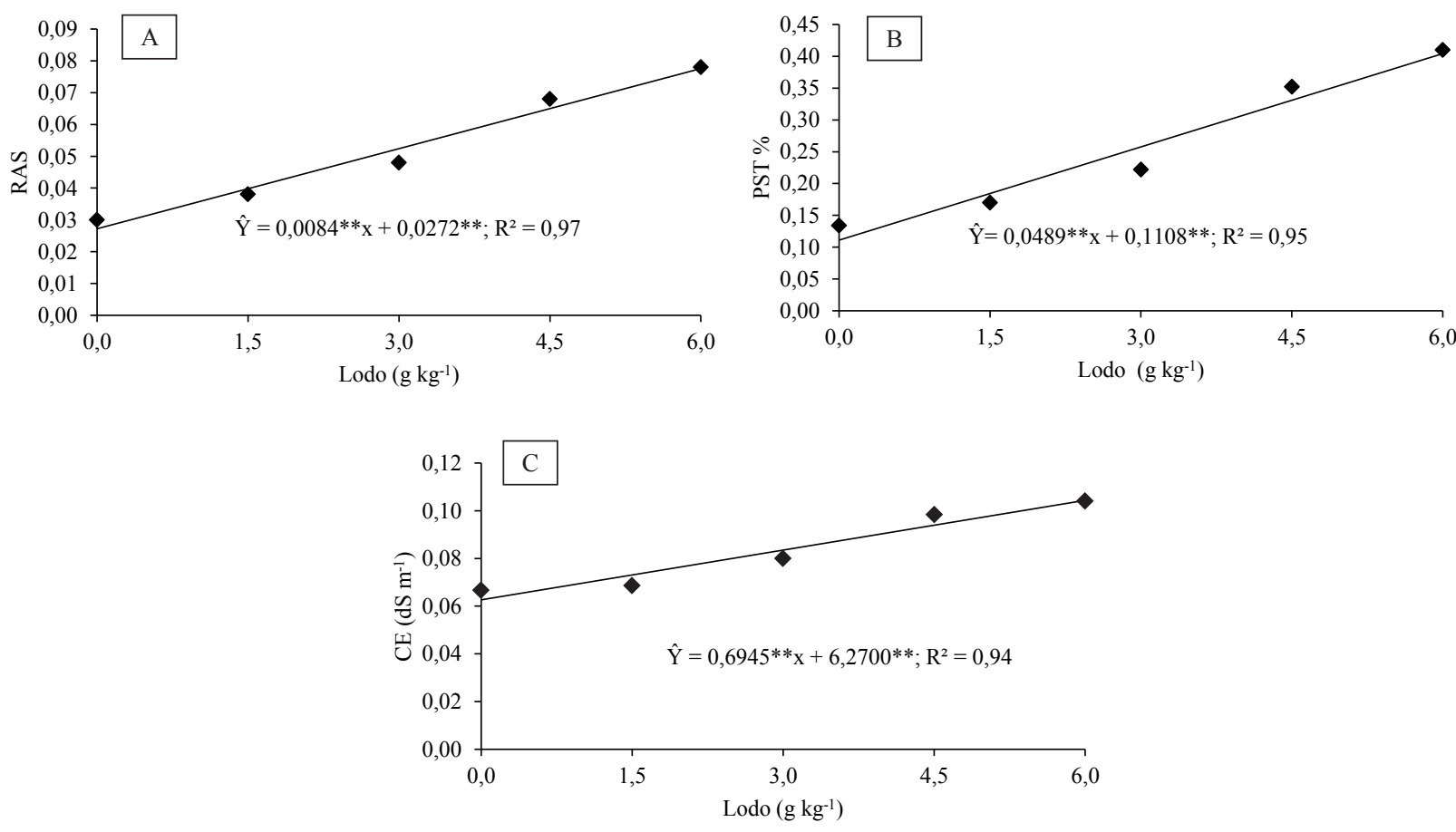

FIGURA 3: Relação da Razão de Adsorção de Sódio - RAS (A), Porcentagem de Sódio Trocável - PST (B) e Condutividade elétrica - CE (C) em função das doses de lodo do curtimento. ${ }^{* *}$ Coeficientes significativos $\mathrm{p}<0,01$

FIGURE 3: Value of the Sodium Adsorption Ratio - RAS (A), Exchangeable Sodium Percentage - PST (B) and Electrical Conductivity - CE (C) as a function of doses of the tanning sludge. ** Significant coefficients $\mathrm{p}<0.01$. 
cia com aqueles encontrados por Aquino Neto e Camargo (2000), Costa et al. (2001), Konrad e Castilhos (2002), Martines (2005), Souza et al. (2006), Teixeira et al. (2006).

\section{Influência do lodo de curtimento nas mudas de paricá}

Quanto aos parâmetros morfológicos analisados nas mudas de paricá, altura $(\mathrm{H})$, diâmetro do colo (DC), número de folhas (NF), comprimento da raiz principal (CR), massa seca da parte aérea (MSPA), massa seca da raiz (MSRA), massa seca total (MST), as relações entre essas variáveis MSPA/MSRA; H/DC; H/MSPA, e o índice de qualidade de Dickson IQD (DICKSON et al., 1960), não diferiram com os tratamentos, ou seja, as doses de lodo utilizadas não foram suficientes para superar a adubação convencional. Entretanto, as mudas de paricá tiveram bom desenvolvimento e estavam visivelmente sadias, fato comprovado pelas médias de $\mathrm{H}, \mathrm{DC}, \mathrm{NF}$ e CR, respectivamente, $66,16 \mathrm{~cm}$, $10,76 \mathrm{~mm}, 9$ unid. e $37,40 \mathrm{~cm}$ de todos tratamen- tos. Da mesma maneira, Araújo et al. (2006) não detectaram diferenças entre os tratamentos NPK e as doses de lodo de curtume + PK + inoculação para a espécie madereira Prosopis juliflora (algaroba) na altura, diâmetro do caule e biomassa seca.

Com relação ao aspecto nutricional do paricá, foram observados apenas efeitos dos tratamentos nos teores de $\mathrm{K}$, Na e $\mathrm{S}$ (Figura 4). Ferreira et al. (2003) também não encontraram diferenças com relação aos teores de $\mathrm{Ca}, \mathrm{P}$ e $\mathrm{N}$ na biomassa seca das culturas do milho e soja, entre os tratamentos com NPK + calcário, lodo de curtume + PK e cromo mineral + lodo de curtume + PK em um Argissolo Vermelho distrófico típico.

$\mathrm{O}$ teor de $\mathrm{Cr}^{3+}$ na planta não foi detectado pelo aparelho de leitura, possivelmente pelo fato das quantidades do elemento na planta serem muito baixas. A absorção e translocação de $\mathrm{Cr}^{3+}$ nas plantas são consideradas muito baixas, em torno de 0,02 a $1 \mathrm{mg} \mathrm{kg}^{-1}$ (MALAVOLTA, 1980), e variáveis de espécie para espécie, além de estarem diretamente associadas a formas solúveis desse elemento no solo (SOUZA, 2009). Outra justificativa, de acordo com
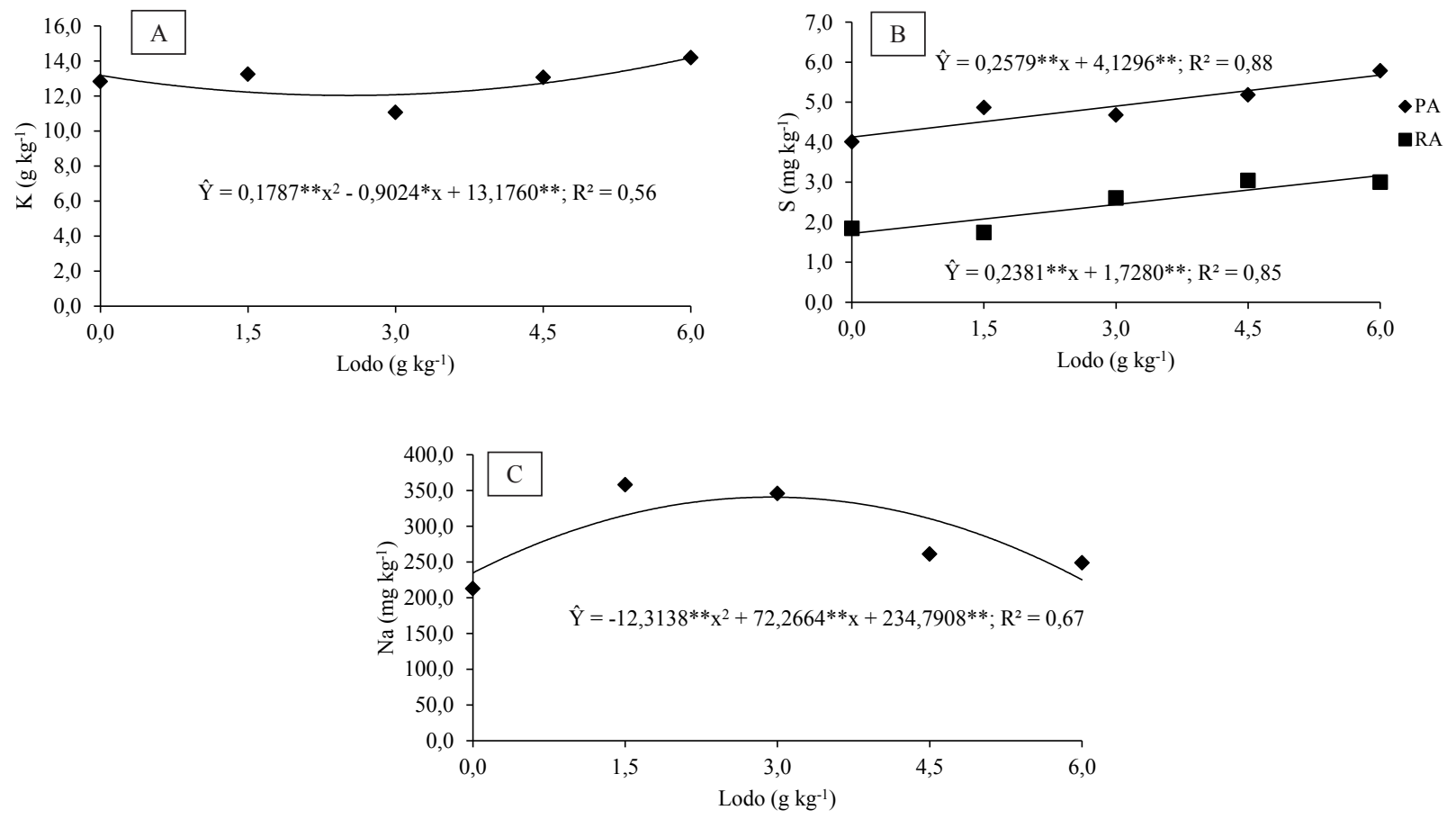

FIGURA 4: Teores de potássio - K (A) e enxofre - $\mathrm{S}(\mathrm{B})$ na parte aérea (PA) e, sódio - Na (C) e enxofre - S na raiz (RA) do paricá em função das doses de lodo do curtimento. **Coeficientes significativos $\mathrm{p}<0,01 ; *$ Coeficientes significativos $\mathrm{p}<0,05$.

FIGURE 4: Content of potassium - K (A) and sulfur - S (B) in shoots (PA), and sodium - Na (A) and sulfur $-\mathrm{S}$ in the root (RA) of paricá in function of doses of the tanning sludge. **Significant coefficients $\mathrm{p}<0.01 ; *$ Significant coefficients $\mathrm{p}<0.05$. 
Macedo e Morril (2008), é que os tecidos radiculares não são capazes de estimular a redução do $\mathrm{Cr}^{3+}$ para $\mathrm{Cr}^{2+}$, forma absorvida pelas plantas.

A absorção de $\mathrm{K}$ pelas mudas de paricá teve efeito significativo apenas na parte aérea, com ajuste quadrático (Figura 4A), contudo, as doses iniciais de lodo do curtimento não foram suficientes para elevar o teor de $\mathrm{K}$ na parte aérea, havendo decréscimo até a dose $2,52 \mathrm{~g} \mathrm{~kg}^{-1}$, correspondendo à absorção de $12,03 \mathrm{~g} \mathrm{~kg}^{-1} \mathrm{de} \mathrm{K}$, ocorrendo aumento a partir desse ponto, sendo que o maior teor de $\mathrm{K}$ foi obtido com a dose $6,0 \mathrm{~g} \mathrm{~kg}^{-1}$ de lodo.

$\mathrm{O}$ fato do $\mathrm{K}$ nas raízes não ter sido significativo pode ter sido influenciado pelo efeito do $\mathrm{Na}$, pois o seu principal papel na nutrição mineral das plantas é substituir o $\mathrm{K}$ em determinadas funções fisiológicas (metabólicas e osmóticas) (KORNDORFER, 2006), tendo em vista a similaridade química entre os dois nutrientes que competem entre si pelos sítios de absorção da raiz (KAWASAKI et al., 1983; ROMERO, 2008). Segundo Marschner (1995), em determinadas espécies, $95 \%$ do K no substrato pode ser substituído pelo $\mathrm{Na}$.

E como pôde ser verificado, somente o teor de Na na raiz (Figura 4A) foi afetado pelas doses estudadas, ajustando-se aos dados de forma quadrática com ponto máximo da curva na dose de $2,93 \mathrm{~g} \mathrm{~kg}^{-1}$, que correspondeu a $340,82 \mathrm{mg} \mathrm{kg}^{-1} \mathrm{de} \mathrm{Na}$, havendo decréscimo a partir deste ponto.

Viana et al. (2001), em experimento com porta-enxertos de videira em solução salina, verificaram que os cultivares IAC 313 e 420-A tiveram restrições na absorção de $\mathrm{K}$ pela raiz em função do aumento de $\mathrm{Na}$ e, conforme aumentava a salinidade, o teor de Na na raiz das cultivares IAC 572, IAC 766 e Ripária do Traviú também aumentava de forma quadrática e linear, demonstrando que essas cultivares eram mais tolerantes, excluindo o $\mathrm{Na}$ das regiões metabolicamente ativas e alocando-o preferencialmente nas raízes e caules. Em graviola, Távora et al. (2004) observaram aumento dos teores de $\mathrm{Na}$ em todas as partes das plantas com o aumento da salinidade, no entanto, houve maior concentração nas raízes das plantas.

Com esse comportamento, o paricá absorveu quantidades significativas de $\mathrm{Na}$ do solo, a fim de que ele não fosse salinizado, sem prejudicar seu desenvolvimento, podendo esta planta ser indicada como fitoextratora de solos contaminados por este elemento.

O aumento do teor de $\mathrm{S}$ no solo, devido às doses de lodo do curtimento, refletiu no acrés- cimo do mesmo nutriente no paricá, tanto na parte aérea quanto na raiz, de forma linear. No entanto, observou-se que as quantidades de $\mathrm{S}$ encontradas na parte aérea foram maiores que as das raízes, porque a assimilação do $\mathrm{S}$ nas folhas é em geral mais ativa do que nas raízes, provavelmente devido ao fato da fotossíntese disponibilizar a ferredoxina reduzida (proteína), e a fotorrespiração gerar a serina (aminoácido), que pode estimular a produção da O-acetilserina, que atua como doadora de elétrons para a redução assimilatória do sulfato (VITTI et al., 2006). Sendo assim, o lodo do curtimento torna-se uma fonte de $\mathrm{S}$ de baixo custo e com elevado potencial nutricional tanto para o solo quanto para a planta.

\section{CONCLUSÕES}

O lodo de curtimento tem potencial corretivo de acidez do solo, com aumento pouco expressivo dos indicadores de salinidade, sodicidade e do teor de $\mathrm{Cr}^{3+}$ disponível.

As características morfológicas das mudas de paricá não foram afetadas pelas doses do lodo do curtimento, não sendo possível otimizar uma dose para a produção de mudas de paricá.

O lodo de curtimento propiciou aumento nos teores de $\mathrm{K}$ e $\mathrm{S}$ na parte aérea e $\mathrm{Na}$ e $\mathrm{S}$ na raiz de paricá.

Os maiores teores de nutrientes no paricá foram obtidos com $6 \mathrm{~g} \mathrm{~kg}^{-1}$ de lodo de curtimento.

Sugerem-se pesquisas com diferentes concentrações de sais no solo, para comprovar o potencial desta espécie em ser fitoextratora em solos contaminados.

\section{AGRADECIMENTOS}

À FAPEMAT pelo financiamento do projeto, à CAPES pela concessão de bolsa estudantil, ao Curso de Pós-Graduação em Agricultura Tropical da Universidade Federal de Mato Grosso e à Empresa Durli Couros Ltda. pela parceria e colaboração no projeto.

\section{REFERÊNCIAS BIBLIOGRÁFICAS}

ALVAREZ , V. H. et al. Determinação e uso do fósforo remanescente. Sociedade Brasileira de Ciência do Solo, Viçosa, v. 25, n. 1, p. 27-33, jan./ mar. 2000. (Boletim Informativo)

AQUINO NETO, V.; CAMARGO, O. A. 
Crescimento e acúmulo de cromo em alface cultivada em dois latossolos tratados com $\mathrm{CrCl}_{3}$ e resíduos de curtume. Revista Brasileira de Ciência do Solo, Viçosa, v. 24, n. 1, p. 225-235, jan./mar.2000.

ARAÚJO, A. S. F. et al. Crescimento e nodulação de mudas de leucena e algaroba em solo com adição de lodo de curtume. Revista Caatinga, UFERSA, v. 19, n. 1, p. 20-24, jan./mar. 2006.

ARAÚJO, F. F. et al. Desenvolvimento do milho e fertilidade do solo após aplicação do lodo de curtume e fosforita. Revista Brasileira de Engenharia Agrícola Ambiental, Campina Grande, v. 12, n. 5, p. 512-516, set./out. 2008.

BARTLETT R.J.; KIMBLE, J. M. Behavior of chromium in soil: I trivalent forms. Journal of Environmental Quality, v. 5, n. 4, p. 379-382, 1976.

BRESSIANI, A. L. Crescimento de mudas de paricá em diferentes saturações por bases e doses de fósforo. 46f. 2010. Dissertação (Mesrado em Agricultura Tropical). Universidade Federal de Mato Grosso, Cuiabá, 2010.

CAMARGO, O. A. et al. Métodos de análise química, mineralógica e física de solos. Campinas: Instituto Agronômico de Campinas, 2009, 77p. (Boletim Técnico, 106)

CAMPOS, M. C. C. et al. Alterações nos atributos físicos e químicos de dois solos submetidos à irrigação com água salina. Revista Caatinga, v. 22, n. 2, p. 61-67, abr./jun. 2009.

CASTILHOS, D. D.; VIDOR, C.; TEDESCO, M. J. Redução do cromo em solo suprido com lodo de curtume e cromo hexavalente. Revista Brasileira de Agrociência, Pelotas, v. 5, n. 3, p. 228-232, set./ dez. 1999.

CETESB - COMPANHIA DE TECNOLOGIA DE SANEAMENTO AMBIENTAL. Lodo de curtume: critérios para o uso em áreas agrícolas e procedimentos para apresentação de projetos (Manual Técnico - Norma P4.233). São Paulo, 1999. 35p.

CETESB - COMPANHIA DE TECNOLOGIA DE SANEAMENTO AMBIENTAL. Valores orientadores para solos e águas subterrâneas no estado de São Paulo (Decisão de diretoria ${ }^{\circ} 195-$ 2005- E, de 23 de novembro de 2005). São Paulo, 2005. 4 p.

COSTA, C. N. et al. Efeito de adição de lodo de curtume sobre as alterações químicas do solo, rendimento de matéria seca e absorção de nutrientes em soja. Revista Brasileira de Agrociência. Pelotas, v. 7, n. 3, p. 189-191, set./dez. 2001.
COSTA, F. S. et al. Estoque de carbono orgânico no solo e emissões de dióxido de carbono influenciadas por

sistemas de manejo no sul do Brasil. Revista Brasileira de Ciência do Solo, v. 32, p. 323-332, 2008.

CRUZ, C. A. F. et al. Efeito de diferentes níveis de saturação por bases no desenvolvimento de mudas de ipê-roxo (Tabebuia impetiginosa (Mart.) Standley). Scientia Forestalis. Piracicaba, n. 66, p. 100-107, dez. 2004.

CRUZ, F. J. R. et al. Mecanismos bioquímicos e fisiológicos da toxidez de alumínio $\left(\mathrm{Al}^{3+}\right)$ em plantas sorgo (Sorghum bicolor (L.) Moench). In: SEMINÁRIO DE INICIAÇÃO CIENTÍFICA/ UFRA， 6.; SEMINÁRIO DE INICIAÇÃO CIENTÍFICA DA EMBRAPA/AMAZÔNIA ORIENTAL, 12., 2008, Belém. Resumos... Belém: UFRA, 2008. 1p.

DICKSON, A. et al. Quality appraisal of white spruce and white pine seedling stock in nurseries. Forest Chronicle, v. 36, p. 10-13, 1960.

EMBRAPA - EMPRESA BRASILEIRA DE PESQUISA AGROPECUÁRIA. Manual de análises químicas de solos, plantas e fertilizantes. 2. ed. Brasília, EMBRAPA Informação Tecnológica, 2009, 627 p.

EMBRAPA - EMPRESA BRASILEIRA DE PESQUISA AGROPECUÁRIA. Produção de Sementes Sadias de Feijão Comum em Várzeas Tropicais: Capacidade de troca de cátions, saturação por bases e saturação por alumínio.2004. Disponível em: http://sistemasdeproducao. cnptia.embrapa.br/FontesHTML/Feijao/ FeijaoVarzeaTropical/solos.htm Acesso em: 05 de set. 2010.

FERREIRA, A. S. et al. Alterações de atributos químicos e biológicos de solo e rendimento de milho e soja pela utilização de resíduos de curtume e carbonífero. Revista Brasileira de Ciência do Solo. Viçosa, v. 27, n. 4, p. 755-763, jul./ago. 2003. FOY, C. D. Soil chemical factors limiting plant root growth. In: HATFIELD, J.L.; STEWART, B.A. Limitations to plant root growth. New York, v. 19, p.97-149, 1992.

KAWASAKI, T.; AKIBA, T.; MORITSUGU, M. Effects of high concentrations of sodium chloride and polyethylene glycol on the growth and ion absorption in plants: I. Water culture experiments in a greenhouse. Plant and Soil, Dordrecht, v. 75, p. 75-85, 1983.

KONRAD, E. E.; CASTILHOS, D. D. Alterações 
químicas do solo e crescimento do milho decorrentes da adição de lodos de curtume. Revista Brasileira de Ciência do Solo. Viçosa, v. 26, n. 1, p. 257-265, jan./mar. 2002.

KORNDÖRFER, G. H. Elementos benéficos. In: FERNANDES, M. S. Nutrição mineral de plantas. Viçosa: Sociedade Brasileira de Ciência do Solo, 2006. p. 355-374.

MACEDO, L. S.; MORRIL, W. B. B. Origem e comportamento dos metais fitotóxicos: revisão de literatura. Tecnologia e Ciência Agropecuária. João Pessoa, v. 2, n. 2, p. 29-38, jun. 2008.

MALAVOLTA, E. Elementos de nutrição mineral de plantas. São Paulo: Agronômica Ceres, 1980. v.1, p. 251.

MAPA - MINISTÉRIO DA AGRICULTURA, PECUÁRIA E ABASTECIMENTO. Laboratório Nacional de Referência Vegetal. Análise de corretivos, fertilizantes e inoculantes: métodos oficiais. Brasília: LANARV, 1988. 104 p.

MARQUES, T. C. L. L. S. M. et al. Exigências nutricionais do paricá (Schizolobium amazonicum, Herb.) na fase de muda. Cerne. Lavras, v. 10, n. 2, p. 167-183, jul./dez. 2004.

MARSCHNER, H. Mineral nutrition of higher plants. 2nd ed. London: Academic Press, 1995. 889 p.

MARTINES, A. M. Impacto do lodo de curtume nos atributos biológicos e químicos do solo. 2005. 74 f. Dissertação (Mestrado em Solos e Nutrição de Plantas) - Curso de Pós-graduação em Agronomia, USP-ESALQ, Piracicaba, 2005.

MARTINES, A. M.; ANDRADE, C. A.; CARDOSO, E. J. B. N. Mineralização do carbono orgânico em solos tratados com lodo de curtume. Pesquisa Agropecuária Brasileira, Brasília, v. 41, n. 7, p. 1149-1155, jul. 2006

MCGRANTH, S. P.; SMITH, S. Chromiumannickel. In: ALLOWAY, B. J. Heavy Metals in Soils. New York: John Wiley\& Sons, 1997. p. 125-147.

PRIEBE, G. P. S. Análise da composição da pele/ couro em função das etapas do seu processamento em curtumes. 2005. 60 f. Trabalho de conclusão de curso (Graduação em Engenharia Química) Universidade Federal do Rio Grande do Sul, Porto Alegre, 2005.

RIBEIRO, A. C.; GUIMARÃES， P. T. G.; ALVAREZ V., V. H. (Ed.). Recomendação para o uso de corretivos e fertilizantes em Minas Gerais: $5^{\text {a }}$ aproximação. Viçosa, Comissão de Fertilidade do Solo do Estado de Minas Gerais - CFSEMG, 1999. $359 \mathrm{p}$.
RICHARDS, L.A. (Ed.) Diagnosis and improvement of saline and alkali soils. Agriculture Handbook. Washington: United StatesSalinity Laboratory, v. 60, p. 160, 1954.

RISTHER, A. Estudo do ciclo do enxofre. Disponível em: http://www.coladaweb.com/ biologia/ecologia/estudo-do-ciclo-do-enxofre Acesso em: 28 dez. 2009.

ROMERO, R. R. Respostas fisiológicas de plantas de Eucalyptus grandis à adubação com potássio ou sódio. 2008. 56 f. Dissertação (Mestrado em Ciências), USP-ESALQ. Piracicaba, 2008.

ROSSA, U. B. Fertilizante de liberação lenta no desenvolvimento de mudas de Paricá. Revista da Madeira, n. 115, jul. 2008.

SALVADOR, J. O. et al. Influência do alumínio no crescimento e na acumulação de nutrientes em mudas de goiabeira. Revista Brasileira de Ciência do Solo, Viçosa, v. 24, n. 4, p. 787-796, out./dez. 2000.

SELBACH, P. A. et al. Descarte e biodegradação de lodos de curtume. Revista do Couro, Estância Velha, v. 4, p. 51-62, jul./ago. 1991.

SOUZA, M. R. F. Uso do lodo de curtume como fonte de nutrientes em plantas forrageiras. 2009. Disponível em: http://www.ufg.br/this2/uploads/ files/66/Milena_Rizzia_1.pdf Acesso em: 03 dez. 2009.

SOUZA, P. H. et al. Influência da saturação por bases do substrato no crescimento e qualidade de mudas Machaerium nictitans (Vell.) Benth. Revista Árvore. Viçosa, v. 32, n. 2, p. 193-201, mar./abr. 2008.

SOUZA, S. M.; CHAVES, L. H. G.; SOUZA, A. P. Efeito da aplicação de lodo de curtume sobre a condutividade elétrica e reação de solo ácido. Agropecuária Técnica, Areia, v. 27, n. 2, p. 77-84, ago./dez. 2006.

TÁVORA, F. J. A. F.; LIMA, E. C. C.; HERNANDEZ, F. F. F. Composição mineral das raízes caules e folhas em plantas jovens de graviola submetidas a estresse salino. Revista Ciência Agronômica, v. 35, n. 1, p. 44-51, jan./jun. 2004. TEIXEIRA, K. R. G. et al. Efeito da adição de lodo de curtume na fertilidade do solo, nodulação e rendimento de matéria seca do caupi. Revista Ciência Agrotécnica, Lavras, v. 30, n. 6, p. 10711076, no./dez. 2006.

VIANA, A. P. et al. Teores de $\mathrm{Na}, \mathrm{K}, \mathrm{Mg}$ e Ca em porta-enxertos de videira em solução salina. Scientia Agricola, Piracicaba, v. 58, n. 1, p. 187191, jan./mar. 2001. 
VITTI, G. C. et al. Cálcio, magnésio e enxofre. M. A. A rapidand precise method for In: FERNANDES, M.S. (Ed.) Nutrição mineral. routinedeterminationoforganiccarbon in soil. Viçosa: Sociedade Brasileira de Ciência do Solo, Communications in Soil Science and Plant 2006. p. 299-325.

YEOMANS, J. C.; BREMMER, J. 1988. 